\title{
Die Zukunft war jetzt
}

Rezension zu Christina Schwenkel (2020): Building socialism. The afterlife of East German architecture in urban Vietnam. Durham: Duke University Press.

Jannik Noeske

Abb. 1 Titel des Buches (Quelle: Duke University Press)

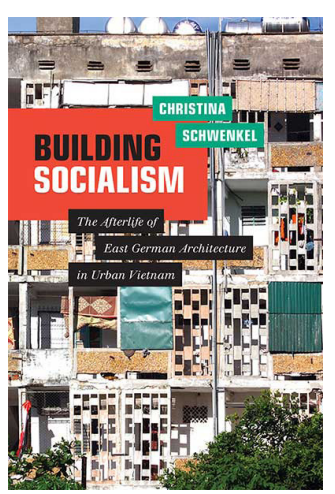

Als das historische Bauhaus 2019 sein 100-jähriges Jubiläum feierte, machte es sich eine Gruppe von Architekt:innen und Kurator:innen zur Aufgabe, den eurozentristischen Fokus der hiesigen Bauhaus-Rezeption um postkoloniale Blicke auf die Modernismen Südostasiens zu erweitern. Nun knüpft das damals entstandene Netzwerk an seine Vorarbeiten an: Unter dem Titel Encounters with Southeast Asian modernism startete im April 2021 eine Reihe von Symposien, die neue Perspektiven auf koloniale und post-koloniale Architekturen im südostasiatischen Raum versprechen. Die Architekturzeitschrift ARCH+, die bereits 2016 zwei ihrer Ausgaben (Heft 226 und 227) Architekturen in Vietnam widmete, liefert dazu ein passendes Schwerpunktheft (Heft 243). Das entstandene Netzwerk mit Projektbeteiligten aus Südostasien, Europa und Nordamerika will Aufmerksamkeit für das Thema generieren, den Architekturdiskurs für Fragen nach Post-Kolonialität in Erbe-Diskussionen sensibilisieren sowie Architektur und Städtebau als „Instrumente der Identitätskonstruktion“ (Ngo 2021:1) aus einer rein architekturhistorischen Betrachtung herausheben.

In der ARCH+-Ausgabe zur „Stillen Avantgarde“ in Vietnam (Heft 226) stellte Christina Schwenkel, Autorin des rezensierten Buches und Teil des Encounters-Netzwerkes, Ansätze ihrer ethnografischen Forschung zur vietnamesischen Stadt Vinh vor (Schwenkel 2016). Die US-amerikanische Kulturanthropologin widmet sich den agencies derjenigen, die an der Zerstörung der Stadt beteiligt waren, besonders aber derjenigen, die an deren Wiederaufbau sowie an der Nutzung und Umnutzung der in den 1970er und 1980er Jahren dort als DDR-Solidaritätsprojekte errichteten Gebäude mitgewirkt haben.

Die Beschäftigung mit den architektonischen und städtebaulichen Hinterlassenschaften der sozialistischen Entwicklungspolitik während des Kalten Kriegs ist vergleichsweise neu. Einen ersten Aufschlag zu einer Bilanz machte die architekturhistorische Zeitschrift The journal of architecture 2012 (Stanek 2012). Das Engagement der DDR in Vietnam kommt hier jedoch nur am Rande vor. Eine aktuellere Zusammenstellung und Interpretation stellte als Ergebnis eines Forschungsprojektes jüngst Andreas Butter 
(2018) vor.[1] Zu erwähnen ist zudem die Dissertation von Dong-Sam Sin (2016), der aus einer Zeitzeugenperspektive den Wiederaufbau zweier nordkoreanischer Städte schildert. Sin hatte in den 1950er Jahren für die ostdeutsche Delegation vor Ort gedolmetscht und er hat nun viele Jahrzehnte später seine Eindrücke der Nachwelt in einer quellengesättigten Dissertation hinterlassen. Auch journalistisch wurde das Thema aufgearbeitet: In ihrem Radiofeature von 2015 zitiert die Journalistin Tanya Lieske aus einem Sansibar-Reiseführer: „Die sansibarische Kooperation mit der damaligen DDR hat im Stadtteil Ng'ombe Plattenbausiedlungen von beträchtlicher Scheußlichkeit hinterlassen. Obwohl der Wasserdruck nicht bis in die oberen Stockwerke reicht und das Flurlicht grundsätzlich nicht funktioniert, ist es für Sansibari schick, hier zu wohnen." (Lieske 2015)

An dieser Stelle setzt die Studie von Christina Schwenkel an: Ist es tatsächlich „schick“, in den DDR-Siedlungen zu wohnen? Im Kontrast zur unerträglich überheblichen Perspektive des in dem Radiofeature zitierten Reiseführers hat sich Schwenkel mehrere Monate in einer Siedlung in Vinh eingemietet und dort mit historiografischen, insbesondere aber ethnografischen Methoden wie Interviews oder teilnehmenden Beobachtungen nach den Perspektiven der Involvierten gefragt. Diese Herangehensweise, die Schwenkel schon in früheren Arbeiten zu Vietnam eingenommen hat, unterscheidet Building socialism von bisherigen Untersuchungen zur städtebaulichen Entwicklungshilfe innerhalb der sozialistischen Staatengemeinschaft. Fragen nach Akzeptanz und Aneignung werden dort in der Regel oberflächlich oder generalisierend beantwortet. Schwenkel hingegen macht gerade die Erfahrungen der Bewohner:innen, die Interaktion, die Aneignung und die kritische Auseinandersetzung mit dem Gebauten zum zentralen Ausgangspunkt ihrer Städtebau-Ethnografie.

Vinh gehörte früher zum sozialistischen Nordvietnam und gilt als die Stadt, die während des Vietnamkriegs am stärksten zerstört wurde. Zudem ist sie die erste und einzige Modellstadt des anschließenden sozialistischen Wiederaufbaus. In drei Abschnitten und zehn Kapiteln bietet Schwenkel zunächst eine Chronologie der Zerstörung der Stadt Vinh, des - geplanten Wiederaufbaus und des Nachlebens des kleinen Wohngebietes Quang Trung in Vinh, das mithilfe von Planer:innen und Wirtschaftshilfen aus der DDR ab 1975 errichtet wurde.

Die methodischen und inhaltlichen Zugänge zu diesen Themen sind vielfältig und sollen hier nicht umfassend wiedergegeben werden. Hervorzuheben ist jedoch die differenzierte Betrachtung relationaler Aspekte des Austauschs. So thematisiert Schwenkel die gegenseitigen Repräsentationen der solidarischen Aufbauhilfe: Wie wurde in Ostdeutschland für das Projekt und um Spenden für Vietnam geworben? Wie funktionierte die Emotionalisierung Vinhs als „Dresden Vietnams“? Wie manifestiert sich bis heute ein spezifisches Ostdeutschland-Bild vor Ort, zum Beispiel über die Markierung „ViệtĐức“(Vietnam-Deutschland), die an gebauten Zeugnissen der Zusammenarbeit angebracht wurde? Schwenkel beleuchtet auch die Versuche, dabei paternalistisch-kolonialistische Abhängigkeiten zu überwinden, konstatiert aber, dass die eingeschriebenen Hierarchien diese Verhältnisse letztendlich nur reproduzieren konnten (Schwenkel 2020: 126).

Auch die Erkenntnis, dass die gebauten Wohnblocks nicht etwa Plattenbauten in ostdeutscher Großtafelbauweise sind, sondern traditionell gemauert 
und verblendet, straft vielleicht das ein oder andere hiesige Klischee Lügen (Schwenkel 2020: 172): Insgesamt versuchten die Ostdeutschen, die Planung an die lokalen Gegebenheiten anzupassen. Aber auch lokale Expert:innen konnten an manchen Stellen eigene Akzente setzen - zum Beispiel eigene Grundrisse verwirklichen. Deshalb differenziert Schwenkel den Begriff des Transfers und schlägt in Anlehnung an Esra Akcan (2012) translation als den passenderen Terminus vor, da schon der Akt des Transports in der Regel eine Übersetzungshandlung bedeute. Schwenkel erweitert dieses Konzept aber noch um den anschließenden Akt des Gebrauchs (Schwenkel 2020: 6): Ob ein Wohngebäude von einer ostdeutschen oder von einer vietnamesischen Brigade gebaut wurde, entschied später auch darüber, wie die Gebäude wahrgenommen und genutzt worden sind.

Dieser hohe Grad der Binnendifferenzierung, mit der Schwenkel Rezeptions- und Aneignungsprozesse innerhalb der vietnamesischen Bewohner:innenschaft schildert, bezieht eine wertvolle und hierzulande leider selten wahrgenommene Perspektive. Ein besonderes Anliegen ist Schwenkel die Repräsentation weiblicher Arbeiterinnen, die aus ländlichen Gebieten in die Stadt gezogen sind. Sie steuerten den größten Teil der Arbeitskraft zum Wiederaufbau bei, leben teilweise bis heute in dem Wohngebiet und sind dort mittlerweile Entwertungserfahrungen und Verdrängungsgefahren ausgesetzt. Dies ist der Schwerpunkt des abschließenden Teils der Studie. Dieser widmet sich der wirtschaftlichen Öffnung und schleichenden Neoliberalisierung Vietnams seit 1986 und beschreibt Prozesse, die die egalitären Ansprüche des Wohnungsbaus aus staatssozialistischer Zeit zu konterkarieren drohen.

Dass Schwenkel ihre Studie um die Thematisierung von Gender und Körper anreichert, sticht besonders hervor. Nicht nur überwindet sie dabei etablierte Modi der Architekturhistoriografie, die in erster Linie männliche, zumeist weiße Planer als die zentralen Akteure positioniert, sondern sie ermöglicht zudem einfühlsame und gewinnbringende Einblicke in die Bedingungen des Wiederaufbaus, aber auch eine differenzierte Perspektive auf die gebauten Ergebnisse. Damit macht Schwenkel ihre Betrachtungen fruchtbar für den post-kolonialen Zugriff auf die städtebauliche Geschichte von Vinh. Als Beitrag zu einer neuen fachöffentlichen Aufmerksamkeit post-kolonialer Architekturdebatten in Südostasien ist Schwenkels Herangehensweise außerordentlich wertvoll.

Besonders lesenswert sind die Abschnitte des Buches, in denen die Autorin von alltäglichen Begegnungen erzählt. In der Einleitung schildert sie beispielsweise, wie sie als Forscherin im Feld wahrgenommen wurde - in Vietnam und in Deutschland (Schwenkel 2020: $12 \mathrm{ff}$.). Die selbstreflexive Verortung ihrer Positionalität - Teil der ethnografischen Wissenschaftsethik - ist bereits ein erster Einblick in die Vorgehensweisen sowie die Wissensproduktion der Autorin. So schildert Schwenkel, wie ein Museumswärter versuchte, ihre Herkunft aus einer Logik des Kalten Krieges heraus zu erraten: Sei sie aus der Sowjetunion? Aus Deutschland? Aus der Tschechoslowakei? Als sie ihm erläuterte, dass sie Amerikanerin sei, wollte er ihr nicht glauben (Schwenkel 2020: 12). Deutsch-Sein, erklärt sie, werde vor Ort mit Tugend (moral goodness) und technologischem Können (technological prowess) verknüpft - ein direktes Ergebnis der Aufbauarbeit, 
die ostdeutsche Expert:innen in Vinh geleistet hätten (Schwenkel 2020: 13). In Ostdeutschland hingegen sei vielmehr ihre nicht-(west-)deutsche Identität ein Türöffner gewesen. Dort habe man ihr Forschungsinteresse als aufrichtig (genuine) und einfühlsam (symapthetic) angesehen (Schwenkel 2020: 17). Weitere Episoden, wie ein zweiwöchiges Architekturstudium in Vinh, von dem Schwenkel sich ein besseres Verständnis des vietnamesischen Hauses versprach (Schwenkel 2020: 265), oder die Formulierung ihrer eigenen Bedenken bezüglich der Regeln der Müllentsorgung während ihrer Feldforschung (Schwenkel 2020: 228), sind als Beobachtungen gewinnbringend in den Fluss der Argumentation eingewoben und bereichern die Studie nicht nur durch ihre narrative Qualität.

Einzig die Ausführungen zu den Zukunftsbildern des DDR-Städtebaus in Vietnam sind aus meiner Sicht stellenweise nicht in letzter Konsequenz ausgearbeitet. Mag die Untersuchung der utopischen Momente des städtebaulichen Solidarprojekts noch einiges an damals Geglaubtem und Gehofftem abbilden, stößt doch Schwenkels Verständnis der rationalisierten Planung als „Techno-Fanatismus“ (techno-fanaticism, 2020: 20) an seine Grenzen. Ging es den sozialistischen Staaten Deutschlands und Vietnams nicht vielmehr darum, materielle und soziale Ziele zu erreichen, als etwa „Traumwelten des Sozialismus" (dreamworlds of socialism, Schwenkel 2020: 108) aufzubauen? Schwenkel selbst erläutert anschaulich, welche handfesten Interessen beim Aufbau des Sozialismus verfolgt und teilweise erreicht wurden. Utopische und post-utopische Imaginationen in Zukunftsentwürfen und Gegenwartshandeln mögen eine zentrale Rolle gespielt haben, ihre transzendentale Überhöhung verwischt aber die bereits in sich kohärente Analyse der Machtverhältnisse und Übersetzungsleistungen, die den Rahmen für Zerstörung, Aufbau, Verfall und Aufwertung darstellten (Schwenkel 2020: 113).

In sich ist das Buch jedoch sehr rund: Schwenkel schreibt in geschliffenem Englisch, die teilweise beiläufige Integration von Klassikern der Theorie (unter anderem Michel Foucault, Walter Benjamin und Susan Sontag) ist meistens schlüssig. Die urban fragments genannten anekdotischen Zwischenkapitel stellen Gedichte, Bilder, Briefe oder Lieder vor, was überaus gewinnbringend ist. Auch die zahlreichen Abbildungen - teilweise einmalige Archivfundstücke oder Schnappschüsse der Autorin - sind sorgsam ausgewählt und stützen die Argumentation ungemein.

Wie wertvoll Building socialism für die Planungsgeschichtsschreibung ist, wird sich in der weiteren Entwicklung post-kolonialer Ansätze innerhalb der Disziplin zeigen. Zuletzt betonte Robert Home (2018), dass Städtebauhistoriographien des Globalen Südens neue theoretische und methodische Ansätze benötigten. Dabei habe der Werkzeugkasten der ethnografischen Forschung schon zur neueren postkolonialen Planungsgeschichte beigetragen. Es geht nach Home (ebd.: 94) darum, empirische Befangenheiten, den theoretischen Eklektizismus (ebd.) sowie eine zumeist weiß und männlich geprägte Planungsgeschichtsschreibung zu überwinden. Genau diesen Anspruch hat Christina Schwenkel auf überzeugende Weise eingelöst. Auch der von Carolin Genz (2020) postulierte Ansatz, ethnografische Forschungen zum Ausgangspunkt für ein holistisches Grundverständnis des Städtischen zu machen, wird Schwenkels Studie vollumfänglich gerecht. Der damit einhergehenden politischen Verantwortung entzieht sich Schwenkel nicht, etwa 
indem sie auf die Aktualität ihrer Forschung angesichts drohender Abrisse und Umsiedlungen verweist.

Letzteres unterstrich sie nochmals bei der Auftaktveranstaltung der eingangs beschriebenen Konferenzreihe Encounters with Southeast Asian modernism. Angesichts des geplanten Stadtumbaus sei nicht nur das architektonische Erbe unmittelbar bedroht. An erster Stelle würden Fragen nach den Lebensentwürfen und Wohnbiografien der Bewohner:innen von Quang Trung aufgeworfen. Hier zeigt sich sehr anschaulich, welche Bedeutung die ethnografische Geschichtsschreibung in aktuellen Erbe-Diskursen einnehmen kann - und welche Ergebnisse die von Genz (2020: 12) geforderte Verortung der Forschung in der politischen Dimension der Positionalität und in den Politiken ethnografischer Repräsentation hervorzubringen vermag.

Die Bauhaus-Universität Weimar unterstützt die Publikation dieses Beitrags durch eine institutionelle Vereinbarung zur Finanzierung von Publikationsgebühren.

\section{Endnoten}

[1] Die Ergebnisse werden in Teilen online im Portal zur DDR-Planungsgeschichte des IRS Erkner (2018) dokumentiert.

\section{Autor_innen}

Jannik Noeske ist Urbanist und forscht zur Städtebau- und Planungsgeschichte im 20. Jahrhundert sowie zu Fragen nach Erbe und Erinnerung.

janniknoeske@gmx.de

\section{Literatur}

Akcan, Esra (2012): Architecture in translation. Germany, Turkey, and the modern house. Durham: Duke University Press.

Butter, Andreas (2018): Showcase and window to the world: East German architecture abroad 1949-1990. In: Planning Perspectives 33/2, 249-269.

Genz, Carolin (2020): Stadt ethnographisch erforschen: Potenziale reflexiver Positionalität. In: sub \urban. zeitschrift für kritische stadtforschung, 8/3, 11-30.

Home, Robert (2018): Global systems foundations of the discipline. Colonial, postcolonial, and other power structures. In: Carola Hein (Hg.), The Routledge handbook of planning history. London/New York: Routledge, 91-106.

IRSErkner-Leibniz-Institutfür RaumbezogeneSozialforschung(2018): Architekturprojekte der DDR im Ausland. Bauten, Akteure und kulturelle Transferprozesse. http:// ddr-planungsgeschichte.de/auslandsprojekte/ (letzter Zugriff am 6.4.2021).

Lieske, Tanya (2015): Sansibar oder die letzte Doktrin. Zwei deutsche Staaten und ihr Kalter Krieg. Feature. Deutschlandfunk, Hörspiel/Hintergrund Kultur, Redaktion: Marcus Heumann, 25.12.2015, 11.05 Uhr, 55:00 Minuten. http://www.deutschlandfunk. de/20151225-sansibar-oder-die-letzte-doktrin-pdf.media.7cb764469c10e9201a1717ca64 637fb8.pdf (letzter Zugriff am 7.4.2021).

Ngo, Anh-Linh (2021): Editorial. In der Moderne heimisch werden. In: ARCH+ 54/243, 1. Schwenkel, Christina (2016): Den globalen Sozialismus aufbauen. Zur Geschichte der DDR-Stadtplanung in Vinh. In: ARCH+ 49/226, 30-41. 
Schwenkel, Christina (2020): Building socialism. The afterlife of East German architecture in urban Vietnam. Durham: Duke University Press.

Sin, Dong-Sam (2016): Die Planung des Wiederaufbaus der Städte Hamhung und Hungnam in Nordkorea durch die DAG-Städtebaubrigade der DDR von 1955-1962. Eine städtebaugeschichtliche Abhandlung aus der Sicht eines Zeitzeugen. Dissertation an der HafenCity Universität Hamburg. https://edoc.sub.uni-hamburg.de//hcu/ volltexte/2017/346/pdf/Dissertation_Sin_27.02.17_ultimate.pdf (letzter Zugriff am 24.6.2020).

Stanek, Łukasz (2012): Introduction: The „second world's“ architecture and planning in the „Third World“. In: The Journal of Architecture 17/3, 299-307. 